\title{
Characteristic of interior crack initiation and early growth for high cycle and very high cycle fatigue of a martensitic stainless steel
}

\author{
Chengqi Sun ${ }^{\mathrm{a}, \mathrm{b}, *}$, Qingyuan Song ${ }^{\mathrm{a}, \mathrm{b}}$, Lingling Zhou ${ }^{\mathrm{a}, \mathrm{b}}$, Xiangnan Pan ${ }^{\mathrm{a}, \mathrm{b}}$ \\ ${ }^{a}$ State Key Laboratory of Nonlinear Mechanics, Institute of Mechanics, Chinese Academy of Sciences, Beijing, 100190, China \\ ${ }^{\mathrm{b}}$ School of Engineering Sciences, University of Chinese Academy of Sciences, Beijing, 100049, China
}

\section{A R T I C L E I N F O}

\section{Keywords:}

Very high cycle fatigue

Crack initiation

Crack growth rate

Grain refinement

Multi-site crack initiation

\begin{abstract}
A B S T R A C T
In this paper, interior crack initiation and early growth are investigated to determine the high cycle and very high cycle fatigue of a martensitic stainless steel by constant and variable amplitude loading fatigue tests. It is shown that the tested material presents a very large fine granular area (FGA) size (72-176 $\mathrm{m})$, and the value of the stress intensity factor range for FGA remains almost constant irrespective of the stress amplitude and the fatigue life. The equivalent crack growth rate in FGA is of the magnitude $10^{-13}-10^{-11} \mathrm{~m} / \mathrm{cyc}$ as determined by the "tree ring" patterns on the fracture surface under variable amplitude loading. Moreover, transmission electron microscopy shows both refined and coarse grains in the extracted samples beneath the FGA, i.e., there is no inevitable relationship between FGA morphology and refined grains beneath the FGA. Additionally, multi-site crack origins are observed under both constant and variable amplitude loadings. This indicates that the crack tends to initiate from the larger inclusion in the highly stressed region for the inclusion-induced fatigue failure.
\end{abstract}

\section{Introduction}

Very high cycle fatigue (VHCF) has drawn considerable attention in recent years [1-5]. For high-strength steels, the crack usually initiates from the interior inclusion, and the fracture surface often presents a fish-eye pattern with morphology of fine granular area (FGA) surrounding the inclusion [6,7]. This is also the case for the high cycle of some high-strength steels with relatively large fatigue life, such as GCr15 [8] and JIS SUJ2 [9]. It is generally considered that the FGA consumes most of the total fatigue life, especially for VHCF [10-12], although it has a very small size that usually ranges from approximately 10 to $80 \mu \mathrm{m}[13,14]$.

Many studies have shown that the stress intensity factor range at the front of the FGA remained almost constant corresponding to the threshold value $\Delta K_{\text {th }}$ of the crack propagation [9,13-15]. Some observations beneath the FGA in the extracted samples indicate that the FGA is composed of a layer of nano-sized grains in high-carbon steel $[16,17]$, medium-carbon steel [18], martensitic stainless steel, and duplex stainless steel [19].

Some methods have also been attempted to measure the crack growth rate in crack initiation and early growth region for VHCF. For example, Stanzl-Tschegg et al. [20] tested the tubular specimens with pre-notch in vacuum and ambient air by ultrasonic axial cycling for martensitic steels containing $12 \%$ chromium. By comparing the fractographic features between the crack growth specimens tested in vacuum and the fatigue specimen with fine granular and smooth areas of internal fish-eye morphology, they showed that the value of the crack growth rate was $10^{-12} \mathrm{~m} / \mathrm{cyc}$ in FGA, $10^{-11} \mathrm{~m} / \mathrm{cyc}$ in the fish-eye region outside the FGA, and $10^{-9} \mathrm{~m} / \mathrm{cyc}$ in the region outside the fisheye. Ogawa et al. [21] quantified the crack growth rate in FGA and fisheye for SUJ2 steel by using the repeated two-step loading test. From the beach marks observed in the fish-eye region, they obtained the value of $\mathrm{d} a / \mathrm{d} N$ as $10^{-7}-10^{-9} \mathrm{~m} / \mathrm{cyc}$. The crack growth rate in the FGA region was estimated as $10^{-13}-10^{-14} \mathrm{~m} / \mathrm{cyc}$ with the assumption that an ODA (i.e., FGA) of similar size as that observed in the constant amplitude test at the higher stress had been formed, and this ODA then increased to the size observed on the fracture surface after the subsequent lower stress without any effect of stress history. Sander et al. [22] used the arrest marks under variable amplitude loading to calculate the fatigue crack growth rate for $34 \mathrm{CrNiMo6}$ steel in the fish-eye region, and the value of $\mathrm{d} a / \mathrm{d} N$ was obtained between $3 \times 10^{-11}$ and $2 \times 10^{-12} \mathrm{~m} /$ cyc. However, they pointed out that no FGA was visible on the fracture surface, although the cracks nucleated predominately at the interior inclusions of the specimen and a fish-eye pattern was formed.

In this work, the interior crack initiation and early growth is further investigated for high cycle and VHCF of a martensitic stainless steel by constant and variable amplitude loading fatigue tests. The aim of the paper is to evaluate the characteristic of interior crack initiation and

\footnotetext{
* Corresponding author. State Key Laboratory of Nonlinear Mechanics, Institute of Mechanics, Chinese Academy of Sciences, Beijing, 100190, China.

E-mail address: scq@lnm.imech.ac.cn (C. Sun).
} 


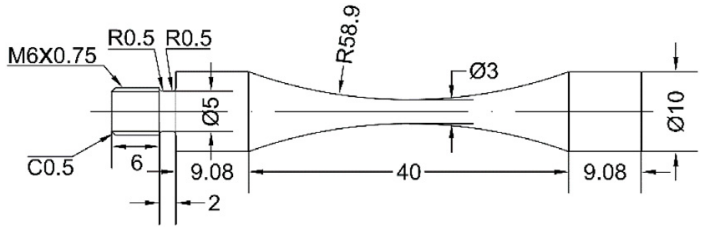

Fig. 1. Specimen shape and dimensions (in $\mathrm{mm}$ ).

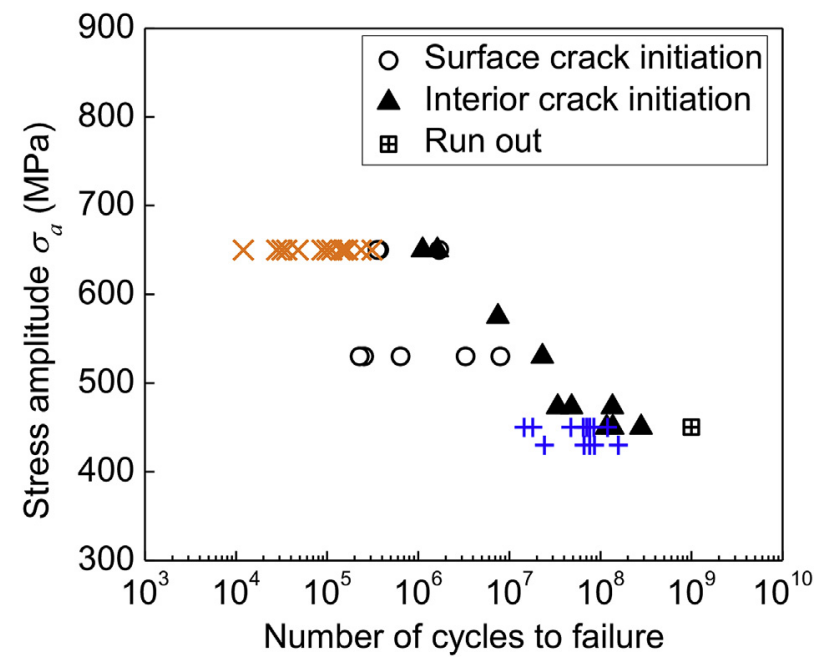

Fig. 2. S-N data for specimens under constant and variable amplitude loadings, in which the symbol "cross" denotes the cumulative cycles under higher stress amplitude and the symbol "plus" denotes the cumulative cycles under lower stress amplitude for the variable amplitude loading.

early growth in high cycle and VHCF regimes depending on the observations made from scanning electron microscopy (SEM) and transmission electron microscopy (TEM) studies.

\section{Materials and methods}

\subsection{Materials}

The material used in this study is a martensitic stainless steel (AISI630) with the chemical compositions $0.047 \mathrm{C}, 15.58 \mathrm{Cr}, 3.65 \mathrm{Ni}$, $3.11 \mathrm{Cu}, 0.74 \mathrm{Mn}, 0.18 \mathrm{Nb}$, and $0.16 \mathrm{Mo}$ in weight percent (Fe balance). Specimens were first heated at $1050^{\circ} \mathrm{C}$ for $60 \mathrm{~min}$ and cooled in air, and then heated again at $850^{\circ} \mathrm{C}$ for $3 \mathrm{~h}$ and oil-quenched; finally, they were tempered for $4 \mathrm{~h}$ at $470{ }^{\circ} \mathrm{C}$ and air-cooled. The tensile strength was $1161 \mathrm{MPa}$, and the yield strength was $1130 \mathrm{MPa}$, the data of which were obtained from two cylindrical specimens of diameter $5 \mathrm{~mm}$ using the servohydraulic test system.

\subsection{Methods}

The fatigue test was conducted on a Shimadzu USF-2000 machine at a resonance of $20 \mathrm{kHz}$ at room temperature in air with pulse of $200 \mathrm{~ms}$ and pause of $200 \mathrm{~ms}$. The stress ratio $R$ was -1 . Compressive cold air was used to cool down the specimens during the fatigue test. The geometry of the specimen is shown in Fig. 1. The notch surface was ground and polished to eliminate the machine scratches before the fatigue test.

Both constant and variable amplitude loadings (repeated two-step loadings) were applied. Variable amplitude loading started from a block of lower stress amplitude $\sigma_{a, L}=450 \mathrm{MPa}$ or $430 \mathrm{MPa}$ with loading cycles $n_{L}=2 \times 10^{6}$ or $3 \times 10^{6}$ and was then followed by a block of higher stress amplitude $\sigma_{a, H}=650 \mathrm{MPa}$ with loading cycles $n_{H}=4 \times 10^{3}$ except for two specimens that experienced first a large number of variable amplitude loadings (starting from a block of lower stress amplitude $400 \mathrm{MPa}$ and followed by a block of higher stress amplitude $650 \mathrm{MPa}$ ). This process was repeated until the specimen failed or up to $10^{9}$ cycles.

The fracture surfaces of the failed specimens were observed by SEM. Further, extracted cross-section samples were first prepared by the focused ion beam (FIB) technique and then observed by TEM with selected area diffraction (SAD) (diffraction area of $680 \mathrm{~nm}$ in diameter). The fracture surfaces of all extracted samples were protected by coating a thin layer of platinum before extraction.

\section{Results and discussion}

\section{1. $S-N$ data}

Fig. 2 shows the S-N data for the specimens under constant and variable amplitude loadings. Detailed loading information for specimens under variable amplitude loading is given in Table 1 . The fatigue data for specimens 1 and 2 experienced at first a large number of variable amplitude loadings, which are not included in Fig. 2. As shown in the figure, for constant amplitude loading, the specimens failed from both the surface and the interior of the specimen under higher stress and failed only from the interior of the specimen under lower stress $(\leq 473 \mathrm{MPa})$. The fatigue life increased with decrease in the stress amplitude for the fatigue failure initiated from the interior of the specimens. For variable amplitude loading, the cumulative cycles (life

Table 1

Loading information of specimens under variable amplitude loading.

\begin{tabular}{|c|c|c|c|c|c|c|}
\hline Specimen No. & $\sigma_{a, L}(\mathrm{MPa})$ & $n_{L}$ (cycle) & $\sigma_{a, H}(\mathrm{MPa})$ & $n_{H}$ (cycle) & Cumulative cycles at $\sigma_{a, L}$ & Cumulative cycles at $\sigma_{a, H}$ \\
\hline $1^{\mathrm{a}}$ & 450 & $2.0 \times 10^{6}$ & 650 & $4.0 \times 10^{3}$ & $2.232 \times 10^{7}$ & $4.4 \times 10^{4}$ \\
\hline $2^{\mathrm{a}}$ & 450 & $2.0 \times 10^{6}$ & 650 & $4.0 \times 10^{3}$ & $1.389 \times 10^{7}$ & $2.4 \times 10^{4}$ \\
\hline 3 & 450 & $2.0 \times 10^{6}$ & 650 & $4.0 \times 10^{3}$ & $1.195 \times 10^{8}$ & $2.4 \times 10^{5}$ \\
\hline 4 & 450 & $2.0 \times 10^{6}$ & 650 & $4.0 \times 10^{3}$ & $8.473 \times 10^{7}$ & $1.7 \times 10^{5}$ \\
\hline 5 & 450 & $2.0 \times 10^{6}$ & 650 & $4.0 \times 10^{3}$ & $7.60 \times 10^{7}$ & $1.5 \times 10^{5}$ \\
\hline 6 & 450 & $2.0 \times 10^{6}$ & 650 & $4.0 \times 10^{3}$ & $7.047 \times 10^{7}$ & $1.4 \times 10^{5}$ \\
\hline 7 & 450 & $2.0 \times 10^{6}$ & 650 & $4.0 \times 10^{3}$ & $1.455 \times 10^{7}$ & $2.8 \times 10^{4}$ \\
\hline 8 & 430 & $2.0 \times 10^{6}$ & 650 & $4.0 \times 10^{3}$ & $1.572 \times 10^{8}$ & $3.1 \times 10^{5}$ \\
\hline 9 & 430 & $3.0 \times 10^{6}$ & 650 & $4.0 \times 10^{3}$ & $8.565 \times 10^{7}$ & $1.1 \times 10^{5}$ \\
\hline 10 & 430 & $3.0 \times 10^{6}$ & 650 & $4.0 \times 10^{3}$ & $7.60 \times 10^{7}$ & $1.0 \times 10^{5}$ \\
\hline 11 & 430 & $3.0 \times 10^{6}$ & 650 & $4.0 \times 10^{3}$ & $2.422 \times 10^{7}$ & $3.2 \times 10^{4}$ \\
\hline 12 & 430 & $3.0 \times 10^{6}$ & 650 & $4.0 \times 10^{3}$ & $6.617 \times 10^{7}$ & $8.8 \times 10^{4}$ \\
\hline 13 & 450 & $1.5 \times 10^{7}$ & 650 & $1.2 \times 10^{4}$ & $1.802 \times 10^{7}$ & $1.2 \times 10^{4}$ \\
\hline 14 & 450 & $1.5 \times 10^{7}$ & 650 & $1.2 \times 10^{4}$ & $6.478 \times 10^{7}$ & $4.8 \times 10^{4}$ \\
\hline 15 & 450 & $1.5 \times 10^{7}$ & 650 & $1.2 \times 10^{4}$ & $4.733 \times 10^{7}$ & $3.6 \times 10^{4}$ \\
\hline
\end{tabular}

${ }^{\text {a }}$ Experiencing at first a number of variable amplitude loadings starting from a block of low-stress amplitude $400 \mathrm{MPa}$ with $1.5 \times 10^{7}$ cycles and then followed by a block of high-stress amplitude $650 \mathrm{MPa}$ with $1.2 \times 10^{4}$ cycles, which is $3.002 \times 10^{8}$ cycles for specimen 1 and $1.501 \times 10^{8}$ cycles for specimen 2 . 

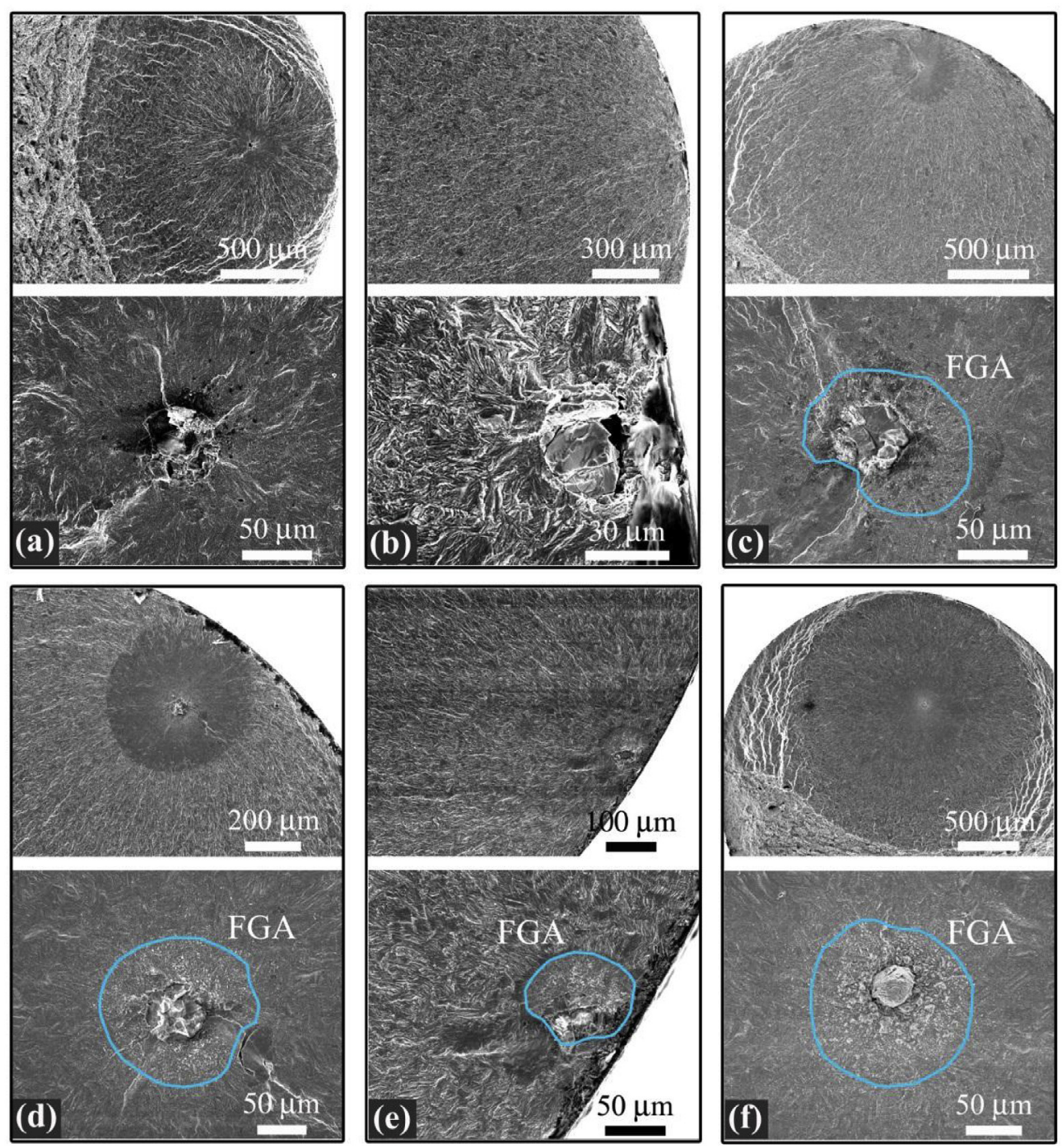

Fig. 3. SEM images for fracture surface of specimens under constant amplitude loading. (a) $\sigma_{a}=650 \mathrm{MPa}, N_{f}=1.114 \times 10^{6}$; (b) $\sigma_{a}=650 \mathrm{MPa}, N_{f}=1.621 \times 10^{6}$; (c) $\sigma_{a}=575 \mathrm{MPa}, N_{f}=7.5 \times 10^{6}$; (d) $\sigma_{a}=473 \mathrm{MPa}, N_{f}=4.816 \times 10^{7}$; (e) $\sigma_{a}=473 \mathrm{MPa}, N_{f}=3.393 \times 10^{7}$; (f) $\sigma_{a}=450 \mathrm{MPa}, N_{f}=1.362 \times 10^{8}$.

Table 2

Fatigue data, inclusion size, and FGA size for the specimens that failed from interior inclusions under constant amplitude loading.

\begin{tabular}{lllll}
\hline Specimen No. & $\sigma_{a}(\mathrm{MPa})$ & $N_{f}$ (cycle) & $a_{\mathrm{In}}(\mu \mathrm{m})$ & $a_{\mathrm{FGA}}(\mu \mathrm{m})$ \\
\hline 1 & 650 & $1.114 \times 10^{6}$ & 47.4 & - \\
2 & 650 & $1.621 \times 10^{6}$ & 31.9 & - \\
3 & 575 & $7.50 \times 10^{6}$ & 47.9 & 95.8 \\
4 & 530 & $2.316 \times 10^{7}$ & 48.6 & 107.3 \\
5 & 473 & $1.353 \times 10^{8}$ & 34.3 & 96.1 \\
6 & 473 & $4.816 \times 10^{7}$ & 54.4 & 136.4 \\
7 & 473 & $3.393 \times 10^{7}$ & 38.9 & 71.9 \\
8 & 450 & $2.804 \times 10^{8}$ & 40.2 & 175.9 \\
9 & 450 & $1.362 \times 10^{8}$ & 33.9 & 129.7 \\
10 & 450 & $1.174 \times 10^{8}$ & 40.2 & 152.7 \\
\hline
\end{tabular}

times) under lower stress amplitude were generally smaller than those under the identical constant amplitude loading. The result for the cumulative cycles under higher stress amplitude was similar compared to the result for those under identical constant amplitude loading. This indicates that higher stress in variable amplitude loading might result in shorter fatigue life for the specimens failed in the VHCF regime. The low stress (below the traditional fatigue limit defined at $10^{7}$ cycles) in variable amplitude loading also might decrease the fatigue life of the specimens failed in high cycle fatigue regime.

\subsection{SEM observation and analyses}

\subsubsection{Constant amplitude loading}

The specimens under constant amplitude loading failed from the surface inclusion/defect or the interior inclusion. For the specimens that failed from the interior inclusion, the fracture surface under stress amplitude $650 \mathrm{MPa}$ presented no FGA morphology, whereas the fracture surface under other stress amplitudes presented FGA morphology. Fig. 3 shows the fracture surfaces of some specimens with and without FGA that failed from interior inclusions.

The inclusion size $a_{\text {In }}$ (square root of the projection area of the inclusion) and FGA size $a_{\mathrm{FGA}}$ (square root of the FGA area including the projection area of inclusion) were measured for specimens failed from interior inclusions, as listed in Table 2. As given in Table 2, the FGA size could be larger than $100 \mu \mathrm{m}$. This differs from the FGA size usually observed for high-strength steels, which is in tens of microns [13,14].

Fig. 4 shows the variation of inclusion size and FGA size on the fatigue life and stress amplitude. As shown in the figure, the inclusion size was independent of fatigue life and stress amplitude, although the FGA size seemed to increase with decrease in stress amplitude or increase in fatigue life.

Here, the variation of stress intensity factor range for FGA (i.e., $\Delta K_{\mathrm{FGA}}$ ) on the fatigue life and stress amplitude was also calculated, and the results are shown in Fig. 5, in which $\Delta K_{\mathrm{FGA}}$ is calculated by 

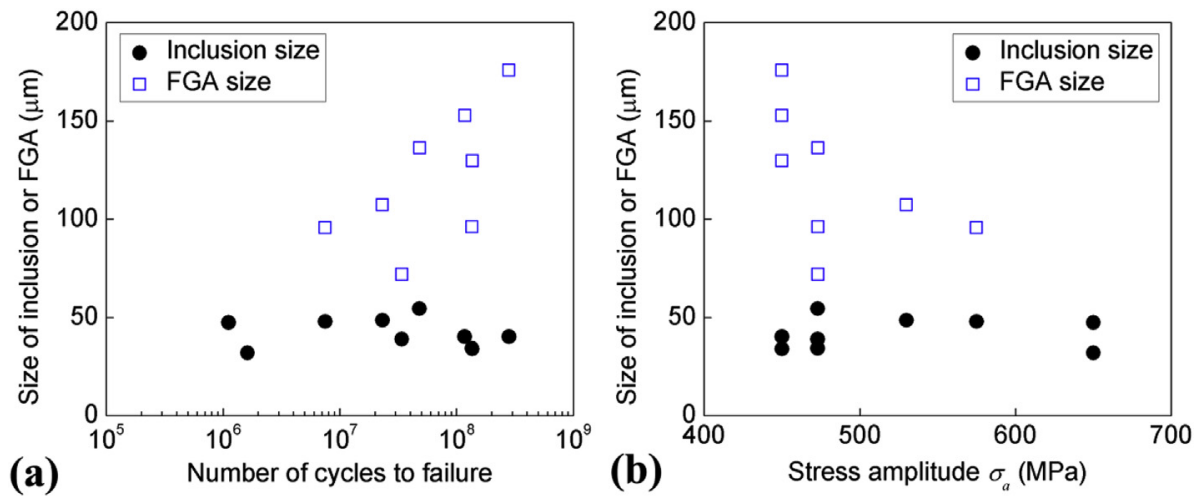

Fig. 4. Variation of inclusion size and FGA size with (a) fatigue life and (b) stress amplitude.
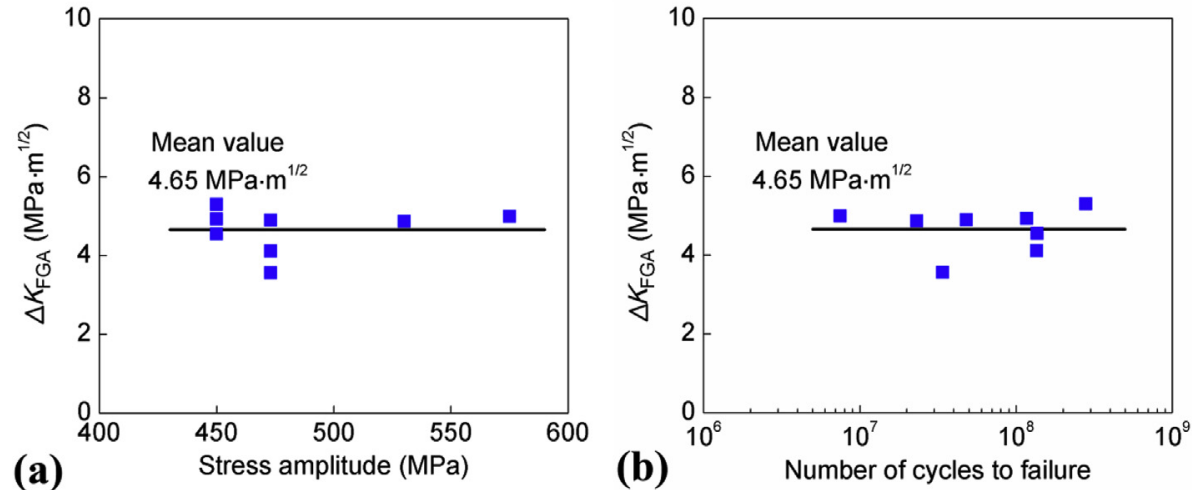

Fig. 5. Variation of $\Delta K_{\mathrm{FGA}}$ with (a) fatigue life and (b) stress amplitude.
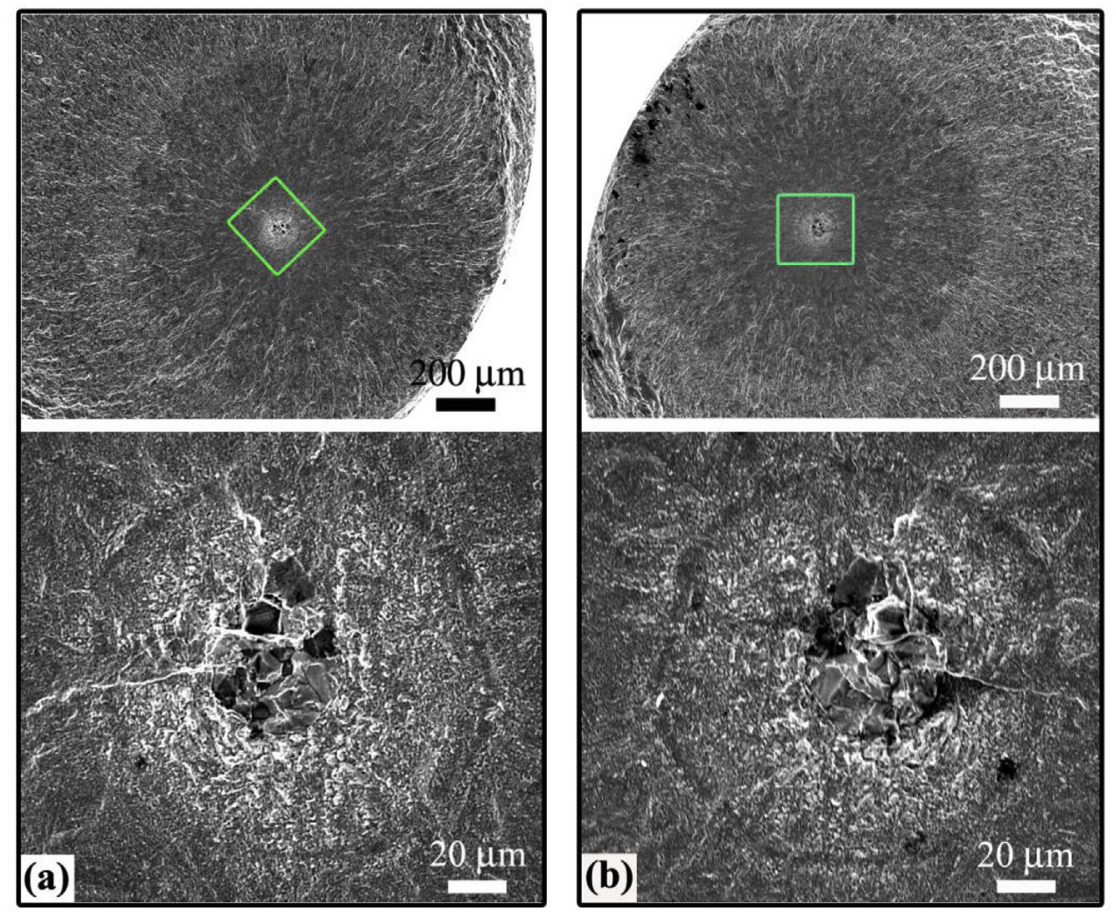

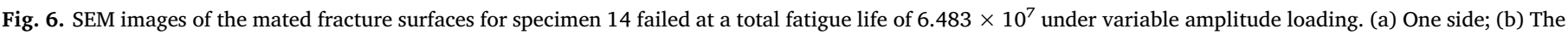
other side.

$\Delta K_{\mathrm{FGA}}=0.5 \sigma_{a} \sqrt{\pi a_{\mathrm{FGA}}}[6,13,23]$. As shown in the figure, the value of $\Delta K_{\mathrm{FGA}}$ remained almost a constant, as reported for the high-strength steels with FGA size in tens of microns [13,14]. The value of $\Delta K_{\mathrm{FGA}}$ was also very close to the threshold value of crack propagation, i.e.,
4.5 MPa $\cdot \mathrm{m}^{1 / 2}$, available for a similar steel (SUS 630) with yield strength $1251 \mathrm{MPa}$ and tensile strength $1368 \mathrm{MPa}$ reported in the literature $[24,25]$. 

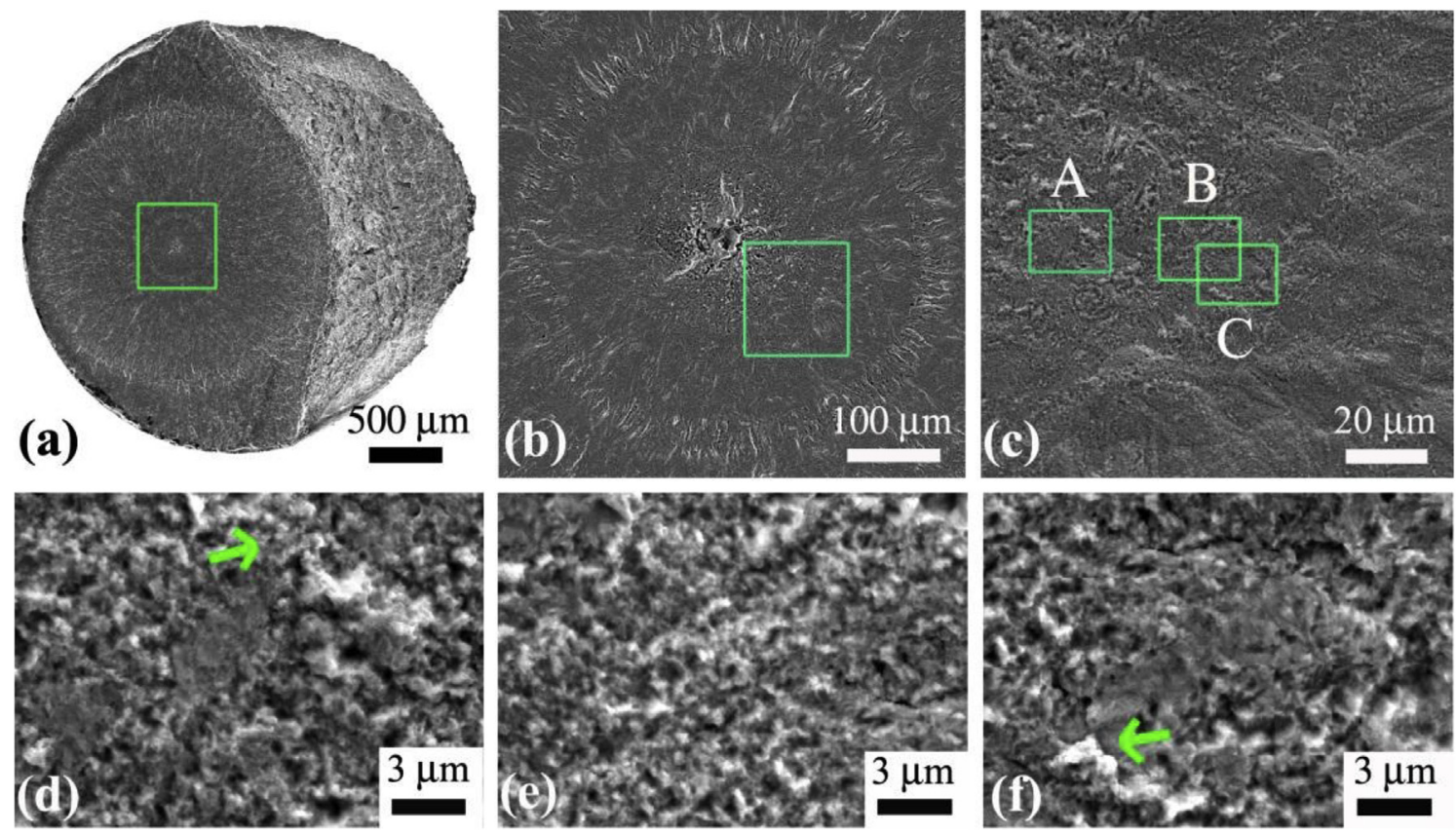

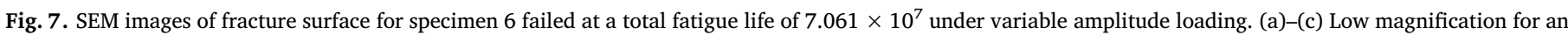

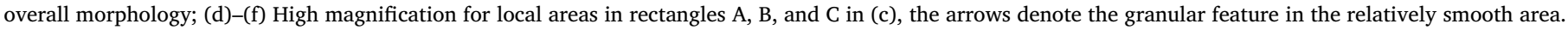
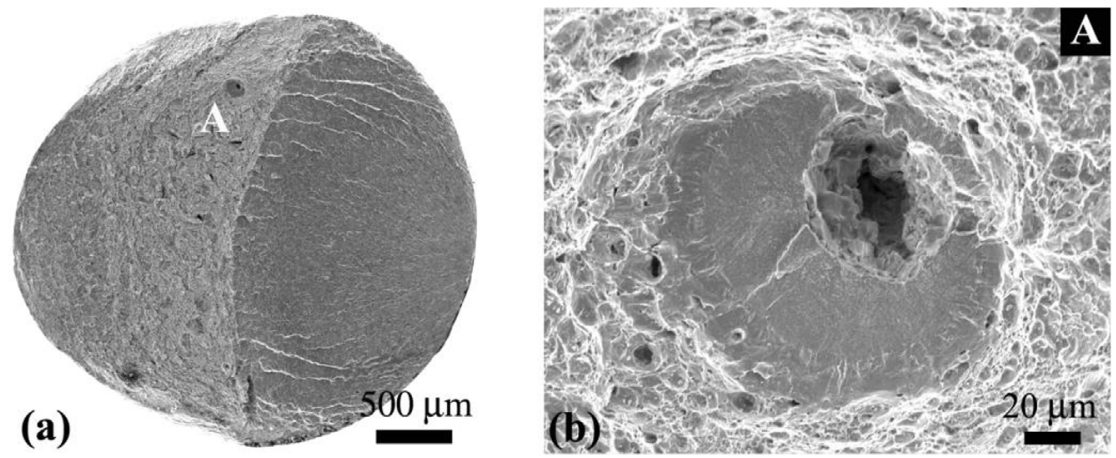

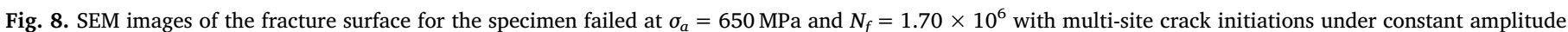
loading. (a) Morphology of the fracture surface; (b) Morphology of secondary crack initiation site.

\subsubsection{Variable amplitude loading}

For variable amplitude loading, all the specimens failed from interior inclusions except one specimen (specimen 7 in Table 1), which failed from surface inclusion. The fracture surface of some specimens presented discernible traces because of the sequence of different cyclic stress amplitudes, as shown in Fig. 6. Based on the result shown in Fig. $4 \mathrm{~b}$ under constant amplitude loading, the specimens under constant stress amplitude of $650 \mathrm{MPa}$ barely presented FGA morphology. Therefore, the smooth annular area between rough areas shown in Fig. 6 corresponds to the loading sequence of the higher stress amplitude $\sigma_{a, H}=650 \mathrm{MPa}$. Fig. 6 indicates that the mated fracture surfaces of failed specimens have very similar morphology, i.e., the rough area or smooth area presents in the mated locations. The observation of the fracture surface for some other failed specimens also shows this result.

Particularly, some fracture surfaces present "tree ring"-like patterns, which paves the way to obtain insights into the involution process of interior crack initiation and early growth. The typical fracture morphology with "tree ring" pattern is shown in Fig. 7. Based on the result shown in Fig. 4b, the rough area (i.e., the granular feature) between relatively smooth areas shown in Fig. $7 \mathrm{c}$ corresponds to the loading sequence of the lower stress amplitude $\sigma_{a, L}=450 \mathrm{MPa}$. Fig. $7 \mathrm{~d}-\mathrm{f}$ shows the magnifications of the local areas for the rough and smooth areas shown in Fig. 7c, respectively. As shown in Fig. 7e, the granular morphology is clear under lower stress amplitude. It was also observed that there is a granular feature in some local regions for the relatively smooth traces, as illustrated in Fig. $7 \mathrm{~d}$ and $\mathrm{f}$. This indicates that the crack extension in crack initiation and early growth stage occurs first in some local and weak regions.

Combined with the variable loading sequence, it takes $2 \times 10^{6}$ or $3 \times 10^{6}$ cycles for the rough area (i.e., the FGA morphology) between relatively smooth ones at the fracture with "tree ring" patterns, while it takes only $4 \times 10^{3}$ cycles for the relatively smooth traces. The widths of the rough and the relatively smooth traces were comparable in "tree ring" patterns, as shown in Fig. $7 \mathrm{~b}$ and c. Compared with the fracture surface with FGA morphology under constant stress amplitude, it could be concluded that the FGA consumes most of the fatigue life in VHCF regime. The "tree ring" patterns at the fracture surface associated with the variable loading sequence also indicate that the formation of the rough area between relatively smooth ones consumes $2 \times 10^{6}$ or $3 \times 10^{6}$ cycles. This suggests that the rough area (i.e., the FGA morphology) could be formed with less than 1 million cycles.

\subsubsection{Multi-sites crack initiation and analyses}

It is observed that some fracture surfaces presented characteristic of 

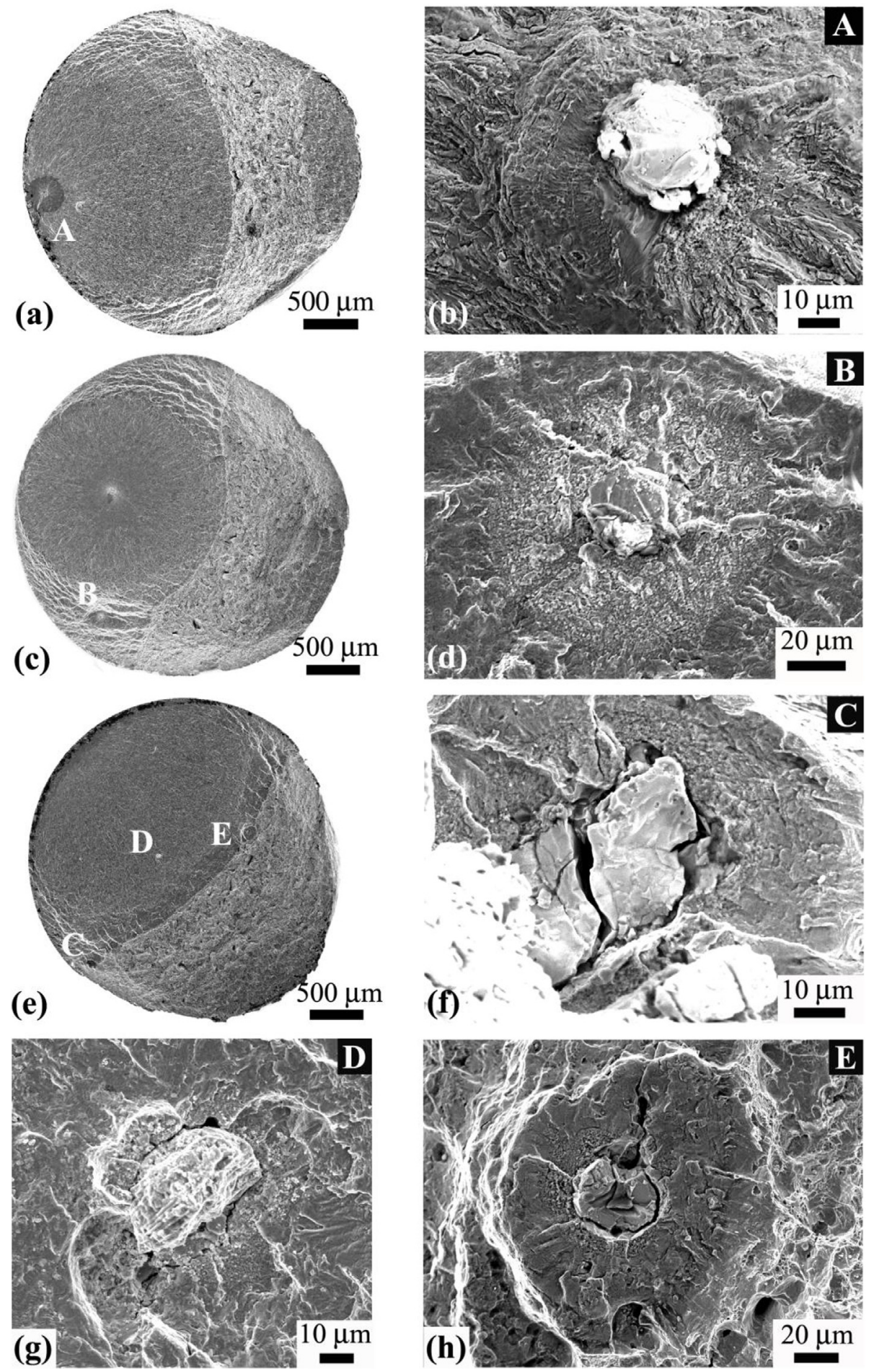

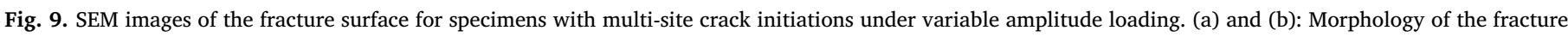

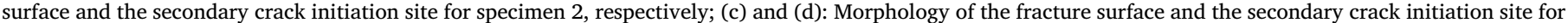
specimens 10, respectively; (e)-(h): Morphology of the fracture surface and the secondary crack initiation sites for specimen 11 , respectively.

multi-site crack initiation. Fig. 8 shows the specimen with surface failure mode and interior secondary crack initiation site under constant amplitude loading. It provides evidence of the competition between surface crack initiation and interior crack initiation in the fatigue of high-strength steels, namely, that the surface failure mode occurs when the fatigue life initiated from the surface is smaller than that initiated from the interior of the specimen; otherwise, the interior failure mode occurs.

Fig. 9 shows the specimens with interior failure mode and secondary crack initiation sites under variable amplitude loading, which provides evidence that there might be multiple crack origins in the specimens. The crack origin that needs the shortest cyclic loadings to result in the fatigue failure will develop to the main crack. Here, the inclusion sizes of the crack origins were also measured and compared for specimens 2 and 10 in Fig. 9(a)-9(d). For specimen 2, the inclusion size was $36.3 \mu \mathrm{m}$ for the main crack origin and $27.0 \mu \mathrm{m}$ for the secondary crack origin. For specimen 10, the inclusion size was $37.5 \mu \mathrm{m}$ for the main crack origin and $29.8 \mu \mathrm{m}$ for the secondary crack origin. Both the inclusion 

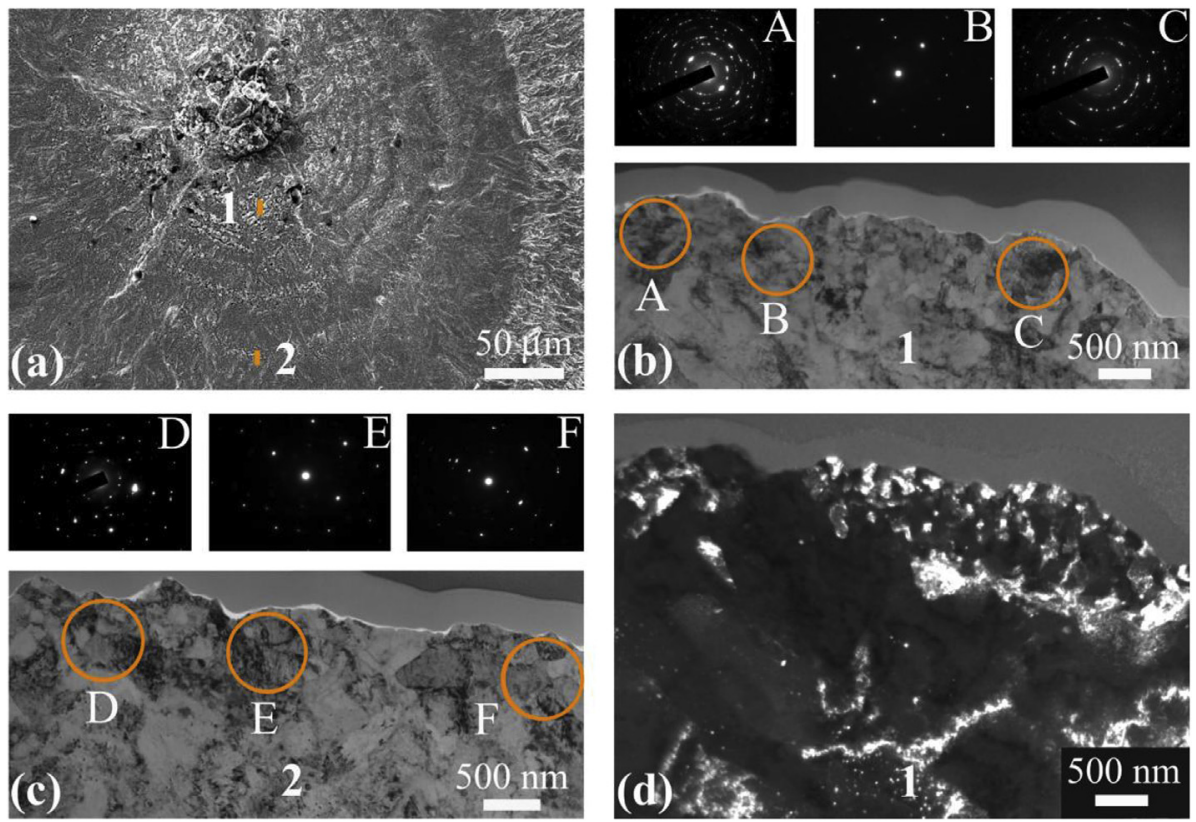

Fig. 10. SEM and TEM images of extracted crosssection samples cut along the loading direction for specimen 9 failed at a total fatigue life of $8.576 \times 10^{7}$ under variable amplitude loading. (a) SEM image of extracted cross-section locations; (b) and (c) Bright-field TEM images of extracted crosssection samples 1 and 2, respectively; the cycles indicate the locations where the SAD diffractions are obtained; (d) Dark-field TEM image of extracted cross-section sample 1 . sizes of the main crack origins were larger than those of the secondary crack origins, hence indicating that the crack tends to initiate from the larger inclusion under the identical loading condition.

\subsection{TEM observation and analyses}

Fig. 10 shows the SEM and TEM images of the extracted cross-section samples from the specimen with "tree ring" pattern under variable amplitude loading. Sample 1 locates in the rough area close to the crack origin (i.e., the inclusion). Sample 2 locates in the rough area by the lower stress amplitude, which is more than $100 \mu \mathrm{m}$ from the crack origin.

It is seen from the TEM image of sample 1 in Fig. 10b, the SAD patterns composed of numerous tiny spots or diffused rings in locations A and C suggest polycrystalline structures consisting of many smaller sized grains (refined grains), while the SAD pattern of isolated spots in location B suggests just a few grains within the diffraction area. The dark field TEM image of sample 1 is given in Fig. 10d, in which the scattered small bright humps close to the fracture surface denote the refined grains. This indicates that the grain refinement regions are discontinuous beneath the rough area (i.e., FGA). The TEM image of sample 3 beneath the rough area by the lower stress amplitude in Fig. 10c also shows that the grain refinement regions are local beneath FGA, i.e., the refined grain does not always exist beneath FGA.

\subsection{Equivalent crack growth rate in FGA}

On the basis of the "tree ring" pattern in the fracture surface, the equivalent crack growth rate in FGA (i.e., crack initiation rate) could be estimated. The cracks after the high- and low-stress sequences are both approximately seen as concentric mode-I internal penny cracks in an infinite solid under a uniform remote tensile stress, and a direction of crack extension with relative clear marks in the SEM image is considered, as illustrated in Fig. 11a, 11c, 11e, 11g, and 11i. The radii of these concentric internal penny cracks are measured along this crack extension direction by using IPP software, as shown in Fig. 11b, 11d, $11 \mathrm{f}, 11 \mathrm{~h}$, and $11 \mathrm{j}$, where $r_{1}, r_{3}, \ldots$ denote the radii of penny cracks after the lower stress sequence and $r_{2}, r_{4}, \ldots$ denote the radii after the higher stress sequence. The values of measured radii are listed in Table 3.

The (equivalent) crack growth rate $\mathrm{d} a / \mathrm{d} N$ and the associated stress intensity factor range $\Delta K$ are calculated by $\left(r_{i+1}-r_{i}\right) / n_{H}$ and
$2 \sigma_{a, H} \sqrt{2\left(r_{i}+r_{i+1}\right) / \pi}$ for the higher stress, and $\left(r_{i+2}-r_{i+1}\right) / n_{L}$ and $2 \sigma_{a, L} \sqrt{2\left(r_{i+1}+r_{i+2}\right) / \pi}$ for the lower stress, respectively, where $i=1,3$, 5. The results are plotted in Fig. 12. It is seen that the crack growth rate in the smooth area is larger than $10^{-10} \mathrm{~m} / \mathrm{cyc}$ (magnitude of crack growth rate corresponding to the traditional crack propagation threshold), whereas the equivalent crack growth rate in the rough area (i.e., FGA morphology under constant stress amplitude) is much lower than $10^{-10} \mathrm{~m} /$ cyc, i.e., the mean crack advance in FGA is far below one lattice spacing per cycle. This indicates that the crack growth in FGA is discrete, i.e., the crack growth in FGA does not extend in all directions in one fatigue cycle, which occurs first in some local regions along the crack extension direction for a number of cycles. This is in agreement with the SEM observation result that there is a granular feature in some little local regions at the smooth traces due to the higher stress amplitude shown in Fig. $7 \mathrm{~d}$ and $\mathrm{f}$.

Considering that the equivalent crack growth rate in the rough area (i.e., FGA) has the tendency to increase with an increase in the stress intensify factor range, an attempt is made to extrapolate the equivalent crack growth rate in FGA close to the inclusion by the linear regression of the measured equivalent crack growth rate in Fig. 12. The inclusions are seen as an internal penny crack with radius $r_{\text {In }}=\sqrt{\operatorname{area}_{\text {In }} / \pi}\left(\operatorname{area}_{\text {In }}\right.$ is the projection area of the inclusion), and the stress intensity factor range for the inclusion is calculated as $4 \sigma_{a} \sqrt{r_{\mathrm{In}} / \pi}$. The inclusion sizes under constant amplitude $\sigma_{a}=450 \mathrm{MPa}$ are used for analysis, the values of stress intensity factor range for which are between 4.44 and $4.84 \mathrm{MPa} \cdot \mathrm{m}^{1 / 2}$. From the results shown in Fig. 12, the equivalent crack growth rate in FGA close to the inclusion might be lower than $10^{-12} \mathrm{~m}$ / cyc. Therefore, the equivalent crack growth rate in FGA might be of the magnitude $10^{-13}-10^{-11} \mathrm{~m} / \mathrm{cyc}$.

\section{Conclusions}

This paper investigates the interior crack initiation and early growth of a martensitic stainless steel in high cycle and VHCF regimes. The main results are summarized as follows:

(1) The FGA size of the present material could be larger than $100 \mu \mathrm{m}$, which differs from the usually observed FGA size in tens of microns for high-strength steels. The value of $\Delta K_{\mathrm{FGA}}$ remains almost a constant independent of the stress amplitude and the fatigue life.

(2) The crack growth in FGA is discrete, which occurs first in some local 

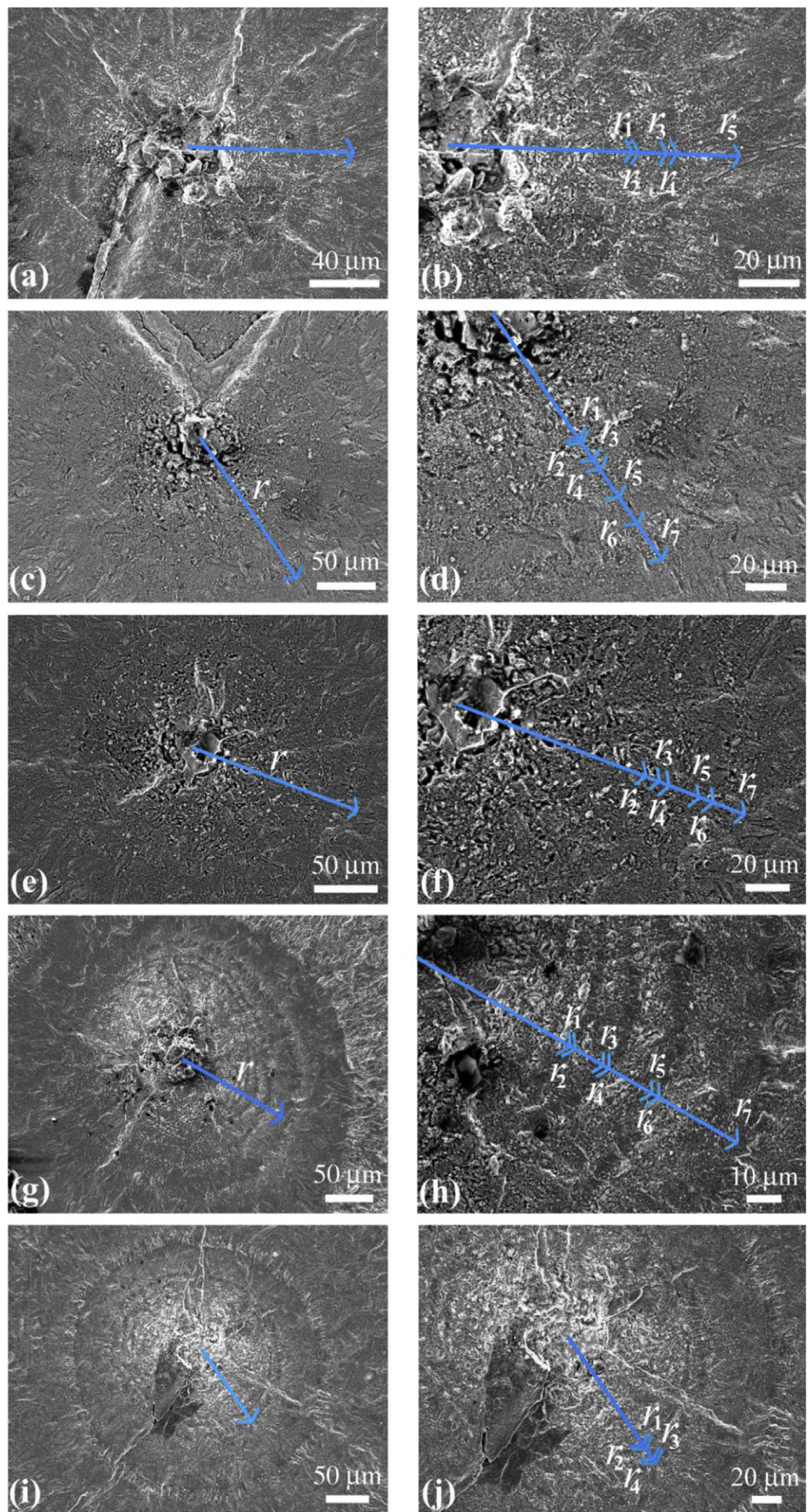

Fig. 11. Images of measurement for radii of penny cracks. (a) and (b): Specimen 2; (c) and (d): Specimen 3; (e) and (f): Specimen 6; (g) and (h): Specimen 9; (i) and (j): Specimen 10.

regions along the crack extension direction for a number of cycles. The equivalent crack growth rate in crack initiation and early growth stage (i.e. FGA) is of the magnitude $10^{-13}-10^{-11} \mathrm{~m} /$ cyc and is in positive correlation with the stress intensity factor range of the initiated cracks.

(3) TEM observation indicates that the refined grain does not always exist beneath FGA, i.e., there is no inevitable relation between the FGA morphology and the grain refinement beneath FGA. 
Table 3

Values of measured radii for penny cracks shown in Fig. 11.

\begin{tabular}{llllll}
\hline Specimen No. & 2 & 3 & 6 & 9 & 10 \\
\hline$r_{1}(\mu \mathrm{m})$ & 60.67 & 74.37 & - & 71.96 & 88.36 \\
$r_{2}(\mu \mathrm{m})$ & 62.99 & 77.25 & 90.84 & 73.47 & 91.55 \\
$r_{3}(\mu \mathrm{m})$ & 72.31 & 87.04 & 97.05 & 82.87 & 99.81 \\
$r_{4}(\mu \mathrm{m})$ & 75.86 & 92.53 & 102.38 & 84.74 & 103.05 \\
$r_{5}(\mu \mathrm{m})$ & 96.3 & 108.72 & 115.81 & 98.84 & - \\
$r_{6}(\mu \mathrm{m})$ & - & 124.22 & 123.05 & 101.04 & - \\
$r_{7}(\mu \mathrm{m})$ & - & 145.13 & 137.96 & 126.62 & - \\
\hline
\end{tabular}

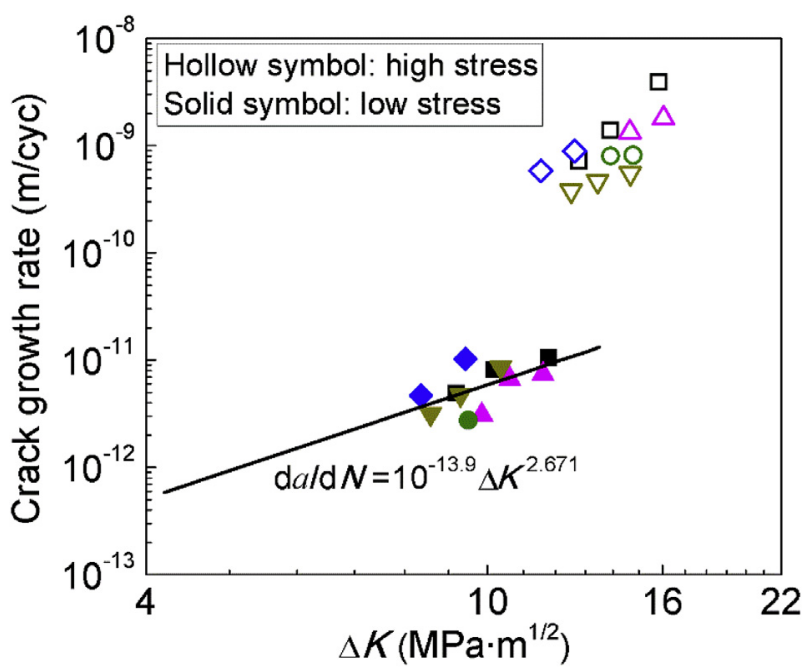

Fig. 12. Crack growth rate versus stress intensity factor range, in which the line denotes the linear regression result of the crack growth rate due to lower stress amplitude.

(4) Multi-site crack initiations are observed at the fracture surfaces of some specimens, which show the competition between the surface crack initiation and interior crack initiation, and the competition for different interior crack initiations. It indicates that, for the inclusion-induced fatigue failure, the crack tends to initiate from the larger inclusion in the highly stressed region.

\section{Acknowledgments}

The authors acknowledge the support of the National Natural Science Foundation of China (91860112), the Innovation Program (237099000000170004), and the Strategic Priority Research Program of the Chinese Academy of Sciences (XDB22020200).

\section{References}

[1] Q.Y. Wang, C. Bathias, N. Kawagoishi, Q. Chen, Int. J. Fatigue 24 (2002) 1269-1274.

[2] J.C. Pang, S.X. Li, Z.G. Wang, Z.F. Zhang, Mater. Sci. Eng. A 564 (2013) 331-341.

[3] H. Matsunaga, C. Sun, Y. Hong, Y. Murakami, Fatigue Fract. Eng. Mater. Struct. 38 (2015) 1274-1284.

[4] B.H. Nie, Z.H. Zhao, D.C. Chen, S. Liu, M.S. Lu, J.L. Zhang, F.M. Liang, Metals 8 (2018) 401.

[5] C. Sun, Q. Song, Y. Hu, Y. Wei, Int. J. Fatigue 117 (2018) 9-12.

[6] Y. Murakami, Metal Fatigue: Effects of Small Defects and Nonmetallic Inclusions, Elsevier Science, Oxford, 2002.

[7] Y. Hong, C. Sun, Theor. Appl. Fract. Mech. 92 (2017) 331-350.

[8] C. Sun, J. Xie, A. Zhao, Z. Lei, Y. Hong, Fatigue Fract. Eng. Mater. Struct. 35 (2012) 638-647.

[9] K. Shiozawa, L. Lu, S. Ishihara, Fatigue Fract. Eng. Mater. Struct. 24 (2001) 781-790.

[10] Z.Y. Huang, D. Wagner, Q.Y. Wang, C. Bathias, Mater. Sci. Eng. A 559 (2013) 790-797.

[11] Y. Hong, Z. Lei, C. Sun, A. Zhao, Int. J. Fatigue 58 (2014) 144-151.

[12] K. Shiozawa, Y. Morii, S. Nishino, L. Lu, Int. J. Fatigue 28 (2006) 1521-1532.

[13] C. Sun, X. Liu, Y. Hong, Acta Mech. Sin. 31 (2015) 383-391.

[14] T. Sakai, J. Solid Mech. Mater. Eng. 3 (2009) 425-439.

[15] A. Zhao, J. Xie, C. Sun, Z. Lei, Y. Hong, Mater. Sci. Eng. A 528 (2011) 6872-6877.

[16] P. Grad, B. Reuscher, A. Brodyanski, M. Kopnarski, E. Kerscher, Scripta Mater. 67 (2012) 838-641.

[17] Y. Hong, X. Liu, Z. Lei, C. Sun, Int. J. Fatigue 89 (2016) 108-118.

[18] Q. Jiang, C. Sun, X. Liu, Y. Hong, Int. J. Fatigue 93 (2016) 352-362.

[19] M.W. Tofique, J. Bergström, K. Svensson, Int. J. Fatigue 100 (2017) 238-250.

[20] S.E. Stanzl-Tschegg, B. Schonbauer, Procedia Eng 2 (2010) 1547-1555.

[21] T. Ogawa, S.E. Stanzl-Tschegg, B.M. Schönbauer, Eng. Fract. Mech. 115 (2014) 241-254.

[22] M. Sander, T. Müller, J. Lebahn, Int. J. Fatigue 62 (2014) 10-20.

[23] K. Shiozawa, T. Hasegawa, Y. Kashiwagi, L. Lu, Int. J. Fatigue 31 (2009) 880-888.

[24] F. Guo, M. Feng, D. Nie, J. Xu, Md S. Bhuiyan, Y. Mutoh, Acta Mech. Solida Sin. 26 (2013) 584-591.

[25] F. Guo, Failure Criteria Based on the Characteristic Length of Materials, Doctor thesis Shanghai Jiao Tong University, 2013. 